\title{
Diffusion-based Model for Synaptic Molecular Communication Channel
}

\author{
Tooba Khan, Student Member, IEEE, Bilgesu A. Bilgin, Member, IEEE, and Ozgur B. Akan, Fellow, IEEE
}

\begin{abstract}
Computational methods have been extensively used to understand underlying dynamics of molecular communication methods employed by nature. One very effective and popular approach is to utilize a Monte Carlo simulation. Although it is very reliable, this method can have a very high computational cost, which in some cases renders the simulation impractical. Therefore, in this paper, for the special case of an excitatory synaptic molecular communication channel, we present a novel mathematical model for the diffusion and binding of neurotransmitters that takes into account the effects of synaptic geometry in three-dimensional space and re-absorption of neurotransmitters by the transmitting neuron. Based on this model we develop a fast deterministic algorithm, which calculates expected value of the output of this channel, namely the amplitude of EPSP (Excitatory Postsynaptic Potential), for given synaptic parameters. We validate our algorithm by a Monte Carlo simulation, which shows total agreement between the results of two methods. Finally, we utilize our model to quantify effects of variation in synaptic parameters such as position of release site, receptor density, size of postsynaptic density (PSD), diffusion coefficient, uptake probability and number of neurotransmitters in a vesicle, on maximum number of bound receptors that directly affect peak amplitude of EPSP.
\end{abstract}

Index Terms-Neuro-spike communication, Synaptic Channel, Diffusion, Receptor Binding, Synaptic variability

\section{INTRODUCTION}

Nanonetworks [1] are envisioned to find significant applications in various disciplines such as health monitoring, intelligent drug delivery systems, quality control of materials and air and water pollution control. Molecular communication [2]-[7], where molecules are used to encode, transmit and receive information, is the most prudent choice of communication paradigm for nanonetworks since it is ubiquitous and performing efficiently in natural processes.

Neuro-spike communication, one of the most evolved and investigated form of molecular communication [8]-[12] , is where electrochemical impulses and neurotransmitters are used for information transfer. Within neuron, information is transferred by electrochemical impulses called action potential $(A P)$ or spike that travel along an axon that branches out at the end and form connections with other neurons. Most of the neurons are physically disconnected from each other having a small gap called synaptic cleft that makes this connection

The authors are with the Next-generation and Wireless Communications Laboratory (NWCL), Department of Electrical and Electronics Engineering, Koc University, Istanbul, 34450, Turkey (e-mail: tkhan15,bilgesubilgin,akan@ku.edu.tr), O. B. Akan is also with the Internet of Everything (IoE) Group, Electrical Engineering Division, Department of Engineering, University of Cambridge, CB3 OFA, UK.

This work was supported in part by the ERC project MINERVA (ERC2013-CoG \#616922), and the EU project CIRCLE (EU-H2020-FET-Open \#665564). more flexible. This connection is commonly known as synapse. The two ends of synapse are pre- and postsynaptic terminals corresponding to transmitter and receiver neurons, respectively as shown in Fig. 1. AP traveling through axon arrives at pre-synaptic terminal and releases neurotransmitters into the synaptic cleft. The major type of neurotransmitter in central excitatory synapses is known as Glutamate. Once released into the cleft, these glutamate molecules are captured by receptors present on postsynaptic terminal generating EPSP. For successive transmissions, neurotransmitters need to be removed from synaptic cleft before next vesicle release. In Central Nervous System, clearance of these neurotransmitters is achieved through diffusion of molecules out of the cleft as well as by re-uptake phenomenon [13], where a fraction of neurotransmitters are re-absorbed by pre-synaptic terminal for re-cycling. Therefore, it is necessary to include the effect of re-absorption to get more realistic model.

There are number of models available for neuronal communication encompassing its biological functionality at various levels of complexity. Integrate-and-fire model [14] is the simplest spiking model that ignores biophysical mechanisms involved in generating spikes. On the other hand, HodgkinHuxley model [15] includes channel conductances, responsible for triggering AP. While addressing the transmission of electrochemical impulses within neuron, these models do not consider biophysical mechanisms involved in interneuronal communication such as vesicle release, diffusion of neurotransmitters, receptor-ligand binding and re-uptake of neurotransmitters. In [8] and [16], physical channel models for neuro-spike communication are proposed that encompasses all of these mechanisms to some extent. The information theoretical analysis of synaptic channel model with trial-totrial variability in EPSP is given in [9] and [17].

All the given models are only close approximations of synaptic channel since capturing complex dynamics of neural signaling into a single model is quite challenging. Therefore, addressing this problem in modular fashion is a feasible solution. Thus, in this paper, our focus is to model diffusion

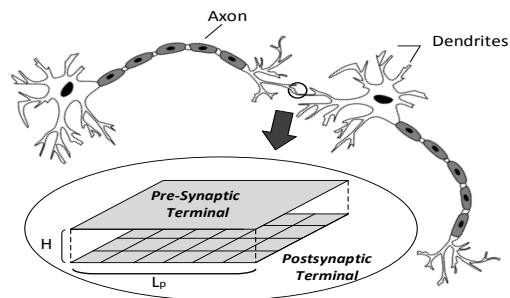

Fig. 1: Geometry of a synaptic channel. 
of neurotransmitters in synaptic cleft and their binding with postsynaptic receptors including the effects of their reflection from pre- and postsynaptic terminals of synaptic channel.

The available models of synaptic channel deal with diffusion of neurotransmitters unrealistically without taking into account all geometrical and statistical determinants. In [8], authors consider diffusion of neurotransmitters in one-dimension, however, authors of [16] consider three-dimensional diffusion model without taking into account their reflections from preand postsynaptic terminals. In [9] and [17], the effect of diffusion is combined with other factors into a single parameter that models trial-to-trial variability in EPSP. In [18]-[20], extensive simulation algorithms are used to determine the position of neurotransmitters within the cleft by drawing trajectories of their motion, however, these models are expensive in terms of time and energy. Therefore, a realistic mathematical model for diffusion and binding of neurotransmitters is required to analyze the effects of different statistical and geometrical parameters on synaptic communication at molecular level.

Thus, in this paper, we consider a synapse in hippocampal region of brain and propose novel mathematical formulation that realistically models diffusion of neurotransmitters and their binding with postsynaptic receptors, incorporating the effects of synaptic geometry in three-dimensional space. Furthermore, we incorporate the effects of pre-synaptic re-uptake of neurotransmitters in our model and validate it through extensive Monte Carlo simulation and existing experimental work. We consider a Single-Input-Single-Output (SISO) synaptic channel as shown in Fig. 1 and, analyze the effects of variation in different synaptic components, namely, vesicle release site, receptor density, PSD size, number of neurotransmitters per vesicle, diffusion coefficient of glutamate and reuptake probability, on EPSP. Consequently, major sources of trial-to-trial variability [21] in single postsynaptic response are discussed. Eventually, this analysis will lead us to understand the mechanism of synaptic plasticity. Finally, we analyze our model of synaptic communication channel by determining error probability in received signal using optimum receiver.

The rest of the paper is organized as follows. In Section II, we give details of physiological assumptions and Monte Carlo simulation, and formulate a mathematical model for diffusion of neurotransmitters and their binding with postsynaptic receptors. Communication theoretical analysis of derived model is performed in Section III. The results are discussed in Section IV. Finally, we conclude this paper in Section V.

\section{SiSO SynAPtic COMMUNiCATION CHANNEL MODEL}

In this section, we propose our model for information transfer across synaptic channel, as shown in Fig. 2. We first describe physiological structure of our channel and make certain realistic assumptions to simplify our analysis. Then, we give mathematical description of molecular processes involved, namely diffusion and ligand-receptor binding, to establish an expected input-output relation for synaptic communication channel. The derived relation forms the basis for a deterministic code, which calculates expected behavior of output of the channel. Later, we briefly present the algorithm of a Monte Carlo simulation that corresponds to mathematical description of the channel previously developed. Both of these descriptions carry out the discussion up to receptor binding process. Afterwards, we give a brief discussion on the time-step, which, as an intrinsic property of the model, is related to the reaction rate constant of ligand-receptor pair. This discussion is an integral part of the descriptions of both the deterministic code and the simulation, as it relates experimentally determined parameter, namely the reaction rate constant, to simulation parameters. Finally, we describe EPSP generation following a given profile of ligand-receptor binding.

\section{A. Physiological Assumptions}

We model synaptic cleft as a rectangular box with height $H$, where top and bottom planes correspond to the pre-synaptic and postsynaptic membranes, respectively, both extending to infinity as in Fig. 1. This assumption does not affect the results since vesicles on pre-synaptic terminal and receptors on PSD are restricted to the limited region on both planes. Moreover, we assume that the movements of neurotransmitters are independent of each other. These assumptions allow us to derive exact analytical solution of diffusion process in the cleft under no-flux boundary conditions, i.e., when there is no neurotransmitter flow across both pre- and postsynaptic membranes. Furthermore, the diffusion model with pre-synaptic reuptake is derived from the former model, i.e., with no-flux boundary conditions, via heuristic arguments.

After vesicle release, diffusion carries neurotransmitters from pre-synaptic end of the cleft to postsynaptic end containing PSD that consists of receptors waiting to capture incoming neurotransmitters to form a ligand-receptor complex. We assume that the receptors are uniformly distributed on PSD, a square-shaped region on the postsynaptic membrane with side length $L_{p}$. PSD has two major kinds of receptors, i.e., AMPA ( $\alpha$-Amino-3-hydroxy-5-methyl-4-isoxazolepropionic acid) and NMDA ( $N$-methyl-D-aspartate) receptors. We suppose that we have only $A M P A$ receptors on PSD since they mediate fast synaptic transmission and contribute in the fast rise in EPSP. NMDA receptors contribute in synaptic plasticity, which is out of the scope of this paper.

\section{B. Mathematical Formulation}

1) Vesicle Release: Vesicles are groups of neurotransmitters enclosed in a thin membrane, which are located right behind the pre-synaptic membrane of the transmitting neuron. Upon the arrival of the AP, depending on the shape of the signal, a number of these vesicles fuse with the pre-synaptic membrane to create a tunnel, through which the transmitters in the vesicles diffuse into the cleft. The region on the pre-synaptic membrane, where this fusion occurs is referred to as the Active Zone (AZ). Since we are considering a central synapse where synaptic boutons contain only one or a few active release zones [17], [22], we can simplify our model by considering that only one vesicle is released in response to an AP.

The arrival of AP at pre-synaptic terminal is a random process owing to the axonal noise. Thus, the channel input $m(t)$ is modeled as an impulse train where probability of receiving an impulse is $P(m=1)=p$. Impulse allows calcium influx into the cell, which triggers vesicle release with probability $P_{r}$. The value of $P_{r}$ depends on the available vesicle pool 


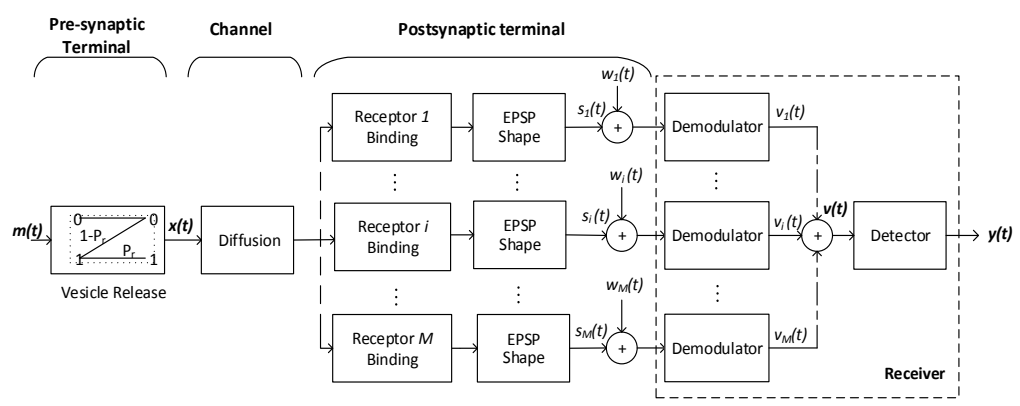

Fig. 2: Block diagram of a synaptic channel.

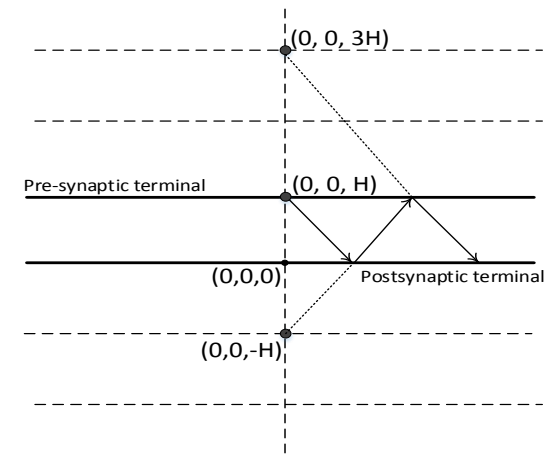

Fig. 3: Diffusion of neurotransmitters in synaptic cleft size and the feedback from postsynaptic terminal signifying receptor saturation [9]. As shown in Fig. 2, vesicle release is represented as a Z-channel, where $1-P_{r}$ is probability of no release even if AP arrives at pre-synaptic terminal.

Once vesicle release starts, it takes very short time for all of the transmitters to enter the cleft, as compared to the total duration of diffusion process in the cleft. Thus, we assume that the contents of the vesicle are released instantaneously and the output of this channel is represented as,

$$
x(t)=N_{0} \delta(t),
$$

where $N_{0}$ is a constant and represents the number of neurotransmitters in one vesicle and Dirac delta represents instantaneous release of the vesicle.

2) Diffusion: Neurotransmitters diffuse into the cleft with a random Brownian motion. The resulting neurotransmitter concentration $C(x, y, z, t)$ is random, and its expected value can be well approximated by Fick's equation:

$$
\frac{\partial C(x, y, z, t)}{\partial t}=D \nabla^{2} C(x, y, z, t)
$$

where $D$ is diffusion coefficient, $t \geq 0$ and $(x, y, z) \in \mathbb{R}^{2} \times$ $[0, H]$. (2) is supplemented with initial distribution provided from the vesicle release module. Assuming that the release occurs at the origin of our $x y$-plane, due to (1) we have

$$
C(x, y, z, 0)=N_{0} \delta(x, y, z-H) .
$$

As for boundary conditions, we first assume that there is no flux of transmitters through pre- and postsynaptic membranes, which corresponds to the Neuman boundary conditions, i.e.,

$$
\frac{\partial}{\partial z} C(x, y, 0, t)=\frac{\partial}{\partial z} C(x, y, H, t)=0 .
$$

Upon integrating (2) over the cleft, one finds that with these boundary conditions, integral of concentration remains constant throughout time, i.e., the expected number of transmitters in the cleft do not change with time.

To find the solution of Fick's equation with initial data as in (3) under the boundary conditions given by (4), we may use symmetry of both the equation and the domain together with the fundamental solution of Fick's equation [23], $c_{\delta}(x, y, z, t)$, in the whole domain $\mathbb{R}^{3}$ given by

$$
c_{\delta}(x, y, z, t)=\frac{N_{0}}{(\sqrt{4 \pi D t})^{3}} e^{\frac{\left(-x^{2}-y^{2}-z^{2}\right)}{4 D t}} .
$$

If there were no boundaries, then solution of (2) with the initial distribution given in (3) would be $c_{\delta}(x, y, z-H, t)$. The no-flux boundary conditions (4) intuitively imply that instead of diffusing freely across boundary, transmitters are reflected back into the cleft. The extra transmitters that will stay in cleft because of this reflection can be accounted for by reflecting the initial distribution across boundaries as in Fig. 3. Then, the distribution of transmitters at any given time is represented by free diffusion with initial distribution taken as sum of all reflections plus original source. As Fick's equation is linear, the corresponding solution will be

$$
\begin{array}{r}
C(x, y, z, t)=2 N_{0} \sum_{k=-\infty}^{\infty} c_{\delta}(x, y, z-(2 k+1) H) \\
=\frac{N_{0}}{(\sqrt{4 \pi D t})^{3}} e^{\frac{\left(-x^{2}-y^{2}\right)}{4 D t}}\left\{2 \sum_{k=-\infty}^{\infty} e^{\frac{-(z-(2 k+1) H)^{2}}{4 D t}}\right\}
\end{array}
$$

Observe that the coefficient 2 in (5) is due to the fact that the source sits right at pre-synaptic boundary, which causes the first reflection from the pre-synaptic side to coincide with the original source. By the symmetries of the equation and the domain, one can easily verify that this solution, restricted to the domain at hand, in fact solves Fick's equation and validates both the initial conditions (3) and the boundary conditions (4).

To model expected concentration of transmitters in presence of pre-synaptic re-uptake, we follow same arguments as in no-flux case with sole difference that we modify reflection coefficient of pre-synaptic boundary $(z=H)$ according to the uptake probability, denoted by $P_{u}$ in units uptake per hit. This means if $P_{u}=0$, no transmitter that reaches the presynaptic membrane is absorbed, while $P_{u}=1$ means that all particles that reach pre-synaptic terminal in the course of time, are absorbed. After making the corresponding modification to include $P_{u}$ in (5), the new solution is obtained as

$$
\begin{aligned}
C_{u}(R, t)= & \frac{N_{0}}{(\sqrt{4 \pi D t})^{3}} e^{\frac{\left(-x^{2}-y^{2}\right)}{4 D t}} \\
& \left\{\sum_{k=-\infty}^{-1}\left(2-P_{u}\right)\left(1-P_{u}\right)^{-(k+1)} e^{\frac{-(z-(2 k+1) H)^{2}}{4 D t}}\right. \\
& \left.+\sum_{k=0}^{\infty}\left(2-P_{u}\right)\left(1-P_{u}\right)^{k} e^{\frac{-(z-(2 k+1) H)^{2}}{4 D t}}\right\} .
\end{aligned}
$$


3) Ligand-Receptor Binding: The transmitters, that reach postsynaptic membrane, either get reflected from membrane, or they bind to the receptors in PSD to form a ligand-receptor complex. This binding process is modeled by assuming that AMPA receptors regularly sample a small effective volume $V_{e}$ around them. The receptors are assumed to have only two configurations, i.e., bound state and unbound state. If a receptor is in unbound state and samples at least one transmitter in its $V_{e}$, then it binds to this transmitter.

Glutamate-receptor binding is a reversible process. Glutamate molecules dissociate from receptors and may rebind several times. The effects of rebinding can be observed from the prolonged decaying phase of EPSP, however, in rising phase of EPSP, the effect of unbinding is negligible. Thus, since we are interested in determining the peak of EPSP, we ignore the effects of unbinding process.

We calculate binding probability by first considering that we have only one neurotransmitter in the cleft, the probability of finding that particle inside the effective volume $V_{e}$ around a receptor at time $t$ is given by the integral

$$
P_{e}(t)=\iiint_{V_{e}} C(x, y, z, t) d x d y d z,
$$

where depending on whether we model diffusion without or with pre-synaptic uptake, the function $C$ is given by (5) or (6) with $N_{0}=1$. Note that, as

$$
\iiint_{\mathbb{R}^{2} \times H} C(x, y, z, t) d x d y d z \leq 1, \quad \forall t \geq 0,
$$

with equality holding for all times in case of no uptake, and only at $t=0$ with uptake, we always have that $P_{e}(t) \in[0,1]$.

Owing to the assumption that all particle motions are independent of each other, the random variable $N_{e}(t)$ representing the number of neurotransmitters found inside $V_{e}$ at time $t$ has a binomial distribution with the expected value

$$
E\left[N_{e}(t)\right]=N(t) P_{e}(t),
$$

where $N(t)$ is the expected total number of unbound neurotransmitters in the cleft at time $t$. Now, according to our assumption it is sufficient to have a single neurotransmitter inside $V_{e}$ for a receptor to be in bound state. It follows that the probability that the receptor will remain unbound after a sampling at time $t$ is $\left(1-P_{e}(t)\right)^{N(t)}$, which simply says that the receptor remains unbound if and only if none of the neurotransmitters are inside $V_{e}$. Thus, the binding probability $P_{b}$ for an unoccupied receptor after a sampling at time $t$ is

$$
P_{b}(t)=1-\left(1-P_{e}(t)\right)^{N(t)} \text {. }
$$

Observe that, by (8) we have following approximation of $P_{b}$ valid for large $N$, i.e., large number of transmitters in the cleft,

$$
P_{b}(t)=1-\left(1-\frac{E\left[N_{e}(t)\right]}{N(t)}\right)^{N(t)} \approx 1-e^{-E\left[N_{e}(t)\right]} .
$$

This binding probability can also be thought of as expected neurotransmitter flux through an unoccupied receptor during the instantaneous sampling process at time $t$. As a consequence, the expected availability of the receptor decreases, which can be interpreted as that for the next sampling process we will have a less number of receptors at the same spot, a 'fractional receptor' so to speak. Due to this decrease in expected availability of receptor, binding probability, at the next time-step, also decreases by the same amount; after all binding probability given by (9) was for a 'whole' receptor. Thus, at the $k^{\text {th }}$ sampling process, $k \geq 1$, the binding probability is found by

$$
P_{b}(k \Delta t)=a(k \Delta t)\left[1-\left(1-P_{e}(k \Delta t)\right)^{N(k \Delta t)}\right],
$$

where $\Delta t$ is the duration of each sampling process or simply a time-step and $a \in[0,1]$ is the expected availability of receptor, which, itself, is then adjusted for the next step by

$$
a((k+1) \Delta t)=a(k \Delta t)-P_{b}(k \Delta t) .
$$

For each receptor, the initial condition that accompanies the iterative scheme (11)-(12) is

$$
a(\Delta t)=1,
$$

which corresponds to the assumption that, before the first sampling all the receptors are unoccupied. The total expected number of bindings at each step, $N_{b}$, can be found by adding the binding probabilities of all the receptors

$$
N_{b}(k \Delta t)=\sum_{j=1}^{M_{0}} P_{b, j}(k \Delta t),
$$

where $M_{0}$ is the number of receptors in the PSD. Finally, at each time-step, we update the number of unbound neurotransmitters left in the cleft by the formula

$$
\begin{aligned}
& N((k+1) \Delta t)= \\
& \left.\left[N_{0}-\sum_{i=1}^{k} N_{b}(i \Delta t)\right)\right] \iint_{\mathbb{R}^{2} \times H} C(x, y, z, t) d x d y d z,
\end{aligned}
$$

where $C$ is given by (5) or (6) with $N_{0}=1$. The integral term is the contribution of uptake, and the sum that is subtracted is due to receptor binding. The fact that we subtract the transmitters that are bound before applying the effect of uptake is justified by the assumed mutually independent motions of transmitters and the fact that the bound transmitters cannot be taken up (since we ignore the dissociation), and neither can the transmitters that are taken up bind a receptor.

\section{Monte Carlo Simulation}

In this subsection we briefly present the details of a Monte Carlo simulation for the synaptic communication channel, the mathematical model of which we have given above.

1) Algorithm: A full simulation of a synaptic channel requires simulation of both diffusion and ligand-receptor binding processes simultaneously. As in our model the latter depends only on the existence of a neurotransmitter inside an effective volume $V_{e}$, the only thing to simulate in our case is the location of neurotransmitters in a regular grid. This is done by taking random seeds as input in each time-step for each of the three coordinates of neurotransmitters, using the distribution given in (6) after normalization. The $x$ and $y$-coordinates have Gaussian distribution with mean $=0$ and variance $=2 \mathrm{Dt}$. 
However, $z$-coordinate follows the distribution derived after considering synaptic geometry given by (6).

Similarly, we calculate the position of $M_{0}$ receptors uniformly distributed on PSD that is kept fixed during entire simulation for a particular value of receptor density. We consider effective volume $V_{e}$ around each receptor, where a neurotransmitter-receptor are most likely to bind. In each timestep, we simulate the position of $N_{0}$ neurotransmitters, and all unbound receptors having at least one neurotransmitter inside its $V_{e}$ are considered bound. In next time-step, we subtract number of bound receptors and neurotransmitters from the pool of unbound receptors and neurotransmitters, respectively. D. Time-step

The $100 \%$ binding probability that we assume at each sampling makes the output of our simulation sensitive to the changes in the time-step $\Delta t$. The decrease in the timestep corresponds to the increase of the sampling rate of the receptors, which yields a rise in the binding rate. Thus, the output of our simulation is highly dependent on the time-step $\Delta t$. In what follows, we derive how to choose the correct time step for given effective volume $V_{e}$ and binding rate constant for glutamate-AMPA pair utilized in literature, see Table I.

In our model we assume only two possible states of the receptors, either bound or unbound. They start in an unbound state, and when they bind to a transmitter, they stay bound forever since we are only analyzing the rising phase of the EPSP. This implies that they are memoryless, i.e., given that the receptor is unbound at time 0 , for a fixed concentration of transmitters and any $t, t_{0} \geq 0$ the probability of binding in the time interval $[0, t]$ is the same as a binding happening inside the interval $\left[t_{0}, t_{0}+t\right]$ provided that the receptor is unbound at time $t_{0}$. It follows that the binding probability $\widetilde{P}_{b}$ of the receptor is exponentially distributed in time with some expected binding time, $\tau_{b}$, where $\tau_{b}$ depends on the concentration near the receptor. Consequently, the probability that the receptor binds in the time interval $(t, t+\Delta t)$ provided that it remains unbound until time $t$ is given by

$$
\widetilde{P}_{b}((t, t+\Delta t))=\frac{1}{\tau_{b}} \int_{0}^{\Delta t} e^{-\frac{\tau}{\tau_{b}}} d \tau \approx 1-e^{-\frac{\Delta t}{\tau_{b}}},
$$

where the approximation is due to the assumption that for small $\Delta t$ the transmitter concentration near the receptor is constant and consequently $\tau_{b}$ is constant. From this we deduce that, for a given concentration $C_{t}$ of neurotransmitters near an unbound receptor, on average the receptor captures a neurotransmitter every $\tau_{b}=\tau_{b}\left(C_{t}\right)$ seconds. This corresponds to a binding rate constant of

$$
\kappa_{r}=\frac{1}{C_{t} \tau_{b}}
$$

in units $M^{-1} s^{-1}$. The expected concentration near the receptor $C_{t}$ can be approximated as

$$
C_{t} \approx \frac{E\left[N_{e}(k \Delta t)\right]}{\left|V_{e}\right|}
$$

where $\left|V_{e}\right|$ is the size of the effective volume. Thus, the binding rate constant of the memoryless model reads

$$
\kappa_{r}=\frac{\left|V_{e}\right|}{E\left[N_{e}(k \Delta t)\right] \tau_{b}}
$$

Comparing (13) with (10) we see that the binding probability we have derived in Section II-B is approximately same as that of a memoryless receptor model provided that one has the identity

$$
E\left[N_{e}(k \Delta t)\right]=\frac{\Delta t}{\tau_{b}} .
$$

Plugging this identity into (14) and rearranging we find the desired relation

$$
\Delta t \approx \frac{\left|V_{e}\right|}{\kappa_{r}}
$$

The value of $\Delta t$ in Table 1 is calculated from this relation.

\section{E. Postsynaptic Response}

When a neurotransmitter is bound to a receptor, a channel opens. This allows ionic flux into the cell, changing its membrane potential called postsynaptic potential. The postsynaptic potential of each receptor is modeled as alpha function [24]

$$
s_{i}(t)=h_{i} \alpha(t),
$$

where $\alpha(t)=\frac{t}{t_{p}} \exp \left(1-\frac{t}{t_{p}}\right)$ with $h_{i}$ as the peak amplitude and $t_{p}$ as time to peak of the response of each receptor.

\section{Communication Theoretical Analysis}

We use optimum receiver for our model as designed in [8] that has following two hypothesis for packet detection:

$$
\begin{gathered}
H_{1}: v=\sum_{i} c_{i} h_{i}+n_{i} \\
H_{0}: v=\sum_{i} n_{i}
\end{gathered}
$$

where $v$ is the input to the detector as shown in Fig. $2, h_{i}$ is a random variable due to the randomness in opening of ionic channel once the receptor is bound as well as in the amount of ions that flow into the channel once it is opened. $c_{i}$ and $n_{i}$ for each receptor $i$ are

$$
\begin{gathered}
c_{i}=\int_{T_{T_{n}}}^{T_{n+1}} \alpha^{2}(t) d t \\
n_{i}=\int_{T_{n+1}}^{T_{n}} w_{i}(t) \alpha(t) d t
\end{gathered}
$$

where $w_{i}(t)$ is a synaptic noise that is considered as additive white Gaussian noise and $\left[T_{n}, T_{n+1}\right]$ is the time interval for receiving one packet of data.

The likelihood ratio for optimum detection is,

$$
\Omega=\frac{P\left(v \mid H_{1}\right)}{P\left(v \mid H_{0}\right)}=\frac{\frac{1}{\sqrt{2 \pi \sigma_{1}^{2}}} \exp \left(-\frac{\left(v-\mu_{1}\right)^{2}}{2 \sigma_{1}^{2}}\right)}{\frac{1}{\sqrt{2 \pi \sigma_{0}^{2}}} \exp \left(\frac{-v^{2}}{2 \sigma_{0}^{2}}\right)}
$$

where $v / H_{1}$ is also a Gaussian random variable according to the central limit theorem since $v$ is the sum of many independent and identically distributed (i.i.d) random variables. The threshold value for detection criterion is

$$
t h=\ln \Omega-\ln \frac{\sigma_{0}}{\sigma_{1}}+\frac{\mu_{1}^{2}}{2 \sigma_{1}^{2}}
$$

where

$$
\begin{gathered}
\mu_{1}=E\left[v \mid H_{1}\right]=\sum_{i} c_{i} E\left[h_{i}\right] \\
\sigma_{1}^{2}=\operatorname{Var}\left[v \mid H_{1}\right]=\sum_{i} c_{i} \operatorname{Var}\left[h_{i}\right]+\operatorname{Var}\left[n_{i}\right]
\end{gathered}
$$


TABLE I: Simulation Parameters

\begin{tabular}{lll}
\hline Synaptic cleft height & $H$ & $20 \mathrm{~nm}[25]$ \\
Neurotransmitters in a Vesicle & $N_{0}$ & $3000[26]$ \\
Diffusion coefficient & $D$ & $0.33 \mu \mathrm{m}^{2} / \mathrm{ms}^{[27]}$ \\
Side length of PSD & $L_{p}$ & $0.4 \mu \mathrm{m}$ \\
Receptor Density & {$[A M P A]$} & $500-3000 / \mathrm{m}^{2}[28]$ \\
Number of receptors & $M_{0}$ & {$[A M P A] \times L_{p}^{2}$} \\
Pre-synaptic Re-uptake & $P_{u}$ & $10 \%$ \\
Binding Rate of AMPA & $\kappa_{r}$ & $78 \times 10^{6} \mathrm{M}^{-1} \mathrm{~s}^{-1}[18]$ \\
Dissociation Rate for AMPA & $\kappa_{d}$ & $750 \mathrm{~s}^{-1}[18]$ \\
Mean of $h_{i}$ & $E\left[h_{i}\right]$ & 1 \\
Variance of $h_{i}$ & $V_{\text {ar }}\left[h_{i}\right]$ & $0.6^{2}$ \\
Variance of noise & $V_{\text {ar }}\left[n_{i}\right]$ & 0.01 \\
Effective Volume & $V_{e}$ & $1 \mathrm{~nm} \times 1 \mathrm{~nm} \times 0.5 \mathrm{~nm}$ \\
Time step & $\Delta t$ & $3.85 \mathrm{~ns}$ \\
Simulation time & $T_{p}$ & $100.9 \mu \mathrm{s}$ \\
\hline
\end{tabular}

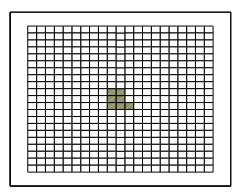

(a) $t=0.5 \mu \mathrm{s}$

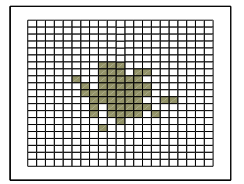

(d) $t=4 \mu \mathrm{s}$

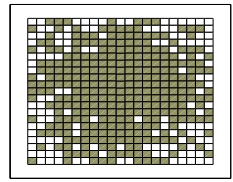

(g) $t=32 \mu \mathrm{s}$

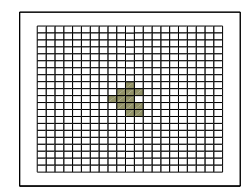

(b) $t=1 \mu \mathrm{s}$

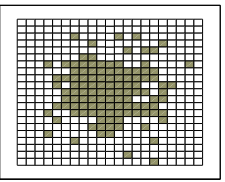

(e) $t=8 \mu \mathrm{s}$

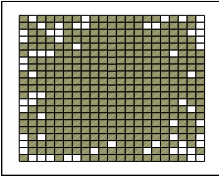

(h) $t=64 \mu \mathrm{s}$

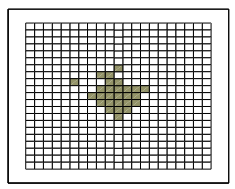

(c) $t=2 \mu \mathrm{s}$

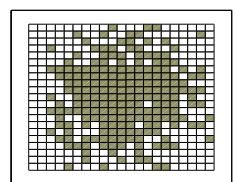

(f) $t=16 \mu \mathrm{s}$

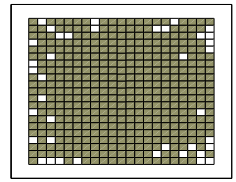

(i) $t=100 \mu \mathrm{s}$
Fig. 4: Time-course of glutamate-receptor binding on $21 \times 21$ grid of receptors with vesicle release site in the center.

$$
\begin{gathered}
\mu_{0}=E\left[v \mid H_{0}\right]=0 \\
\sigma_{0}^{2}=\operatorname{Var}\left[v \mid H_{0}\right]=\sum_{i} \operatorname{Var}\left[n_{i}\right]
\end{gathered}
$$

and the probability of error in the output is given as,

$$
\begin{aligned}
P_{e}= & {\left[P(y<t h \mid x=0)\left(1-P_{r}\right)+P(y<t h \mid x=1) P_{r}\right] } \\
& P(m=1)+P(y>t h \mid x=0) P(m=0)
\end{aligned}
$$

\section{RESUlTS AND Discussion}

\section{A. Determination of EPSP Peak}

The peak of EPSP, as described in Section II-E, directly corresponds to the maximum number of bound receptors, $M_{b, \max }$. Since we neglect the effects of dissociation of transmitters from AMPA receptors, the number of bound receptors, $M_{b}(t)$, obtained from our simulation is a monotonically increasing function of time. Consequently, finding peak time, $T_{p}$ and the peak value, $M_{b, \max }$, seems out of reach. However, we may define these quantities by utilizing dissociation rate constant $\kappa_{d}$, which we have ignored so far. We choose $T_{p}$ such that at $t=T_{p}$ the total binding and dissociation rates are equal, where the total binding rate, $r_{b}(t)$, at a given time $t$ is the rate of change of $M_{b}(t)$ with respect to time, and the total dissociation rate $r_{d}(t)$ is found by multiplying $\kappa_{d}$ and $M_{b}(t)$

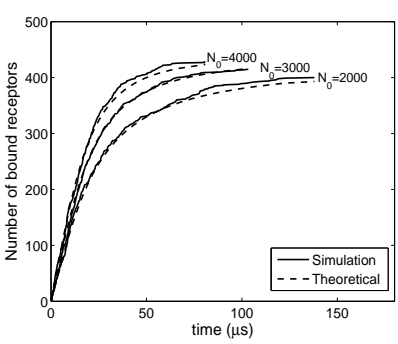

(a) (b)

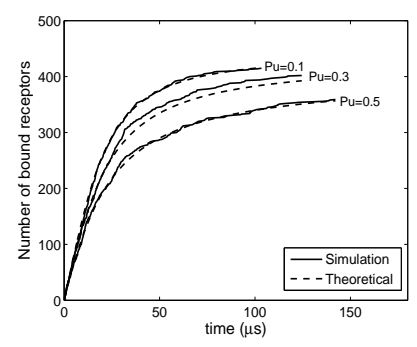

Fig. 5: Time-course of glutamate-receptor binding (a) with $P_{u}=0.1$ (b) with $N_{0}=3000$.

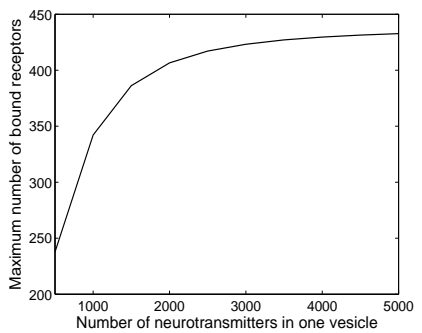

(a)

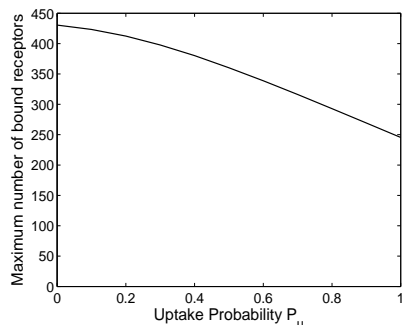

(b)
Fig. 6: Variation in Maximum Number of bound receptors with (a) Number of Neurotransmitters per vesicle (b) Uptake Rate.

at time $t$. We choose $M_{b, \max }$ as the value of $M_{b}(t)$ at $t=T_{p}$. This method of choosing $T_{p}$ and $M_{b, \max }$ exactly corresponds to the assumption that total amount of dissociation from the receptors up until the peak time is negligible.

\section{B. Comparison of two methods}

We perform Monte Carlo simulation described in Section II-C with parameters given in Table I. A square PSD of side length $L_{p}$ is modeled by a $21 \times 21$ grid of small squares with a single receptor in the center of each, i.e. $M_{0}=441$. This corresponds to a receptor density of approximately 2750 receptors $/ \mu^{2}$ falling into the range given in Table I. The time course of glutamate-receptor binding is depicted in Fig. 4. To represent the simulation results, i.e., $M_{b}(t)$ after each timestep, the squares containing bound receptors are highlighted.

It can be observed that the receptors exactly opposite to the vesicle release site are the first to bind due to maximum concentration of neurotransmitters at this point. We see that with these parameters the receptor grid is almost saturated in response to single vesicle release as in Fig. 4. Interestingly, this result is in agreement with the other existing experimental work [29]-[31] as well as the simulation model developed in [18]. The significant difference between the number of neurotransmitters in a vesicle and the receptors present on the PSD assures saturation with single vesicle release.

The results obtained by theoretical model of Section II-B and by simulation algorithm described in Section II-C, are compared in Fig. 5(a) and (b) for different sets of parameters. In Fig. 5(a), the theoretical model is validated for different values of $N_{0}$ and in Fig. 5(b), the theoretical model is validated for different values of uptake probability $P_{u}$. It is observed that the time-course of glutamate-receptor binding from both the methods are similar. This observation signifies the importance of the theoretical model for diffusion and binding of the neurotransmitters developed in this paper, as it is much faster 


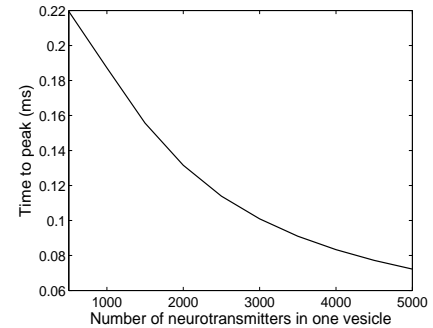

(a)

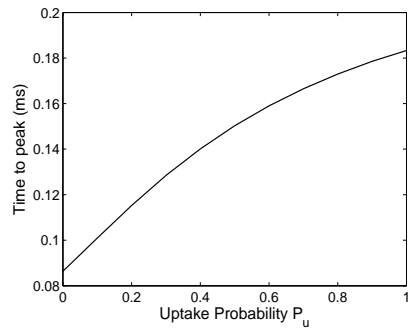

(b)
Fig. 7: Variation in time to peak with (a) Number of Neurotransmitters per vesicle (b) Uptake Rate.

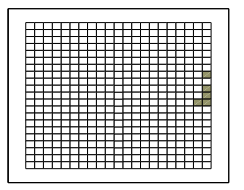

(a) $t=0.5 \mu \mathrm{s}$

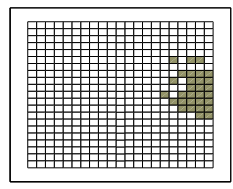

(d) $t=4 \mu \mathrm{s}$

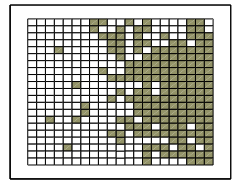

(g) $t=32 \mu \mathrm{s}$

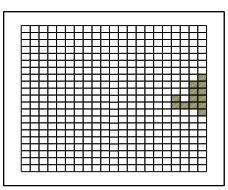

(b) $t=1 \mu \mathrm{s}$

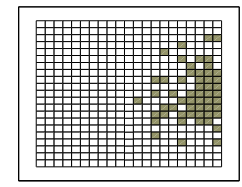

(e) $t=8 \mu \mathrm{s}$

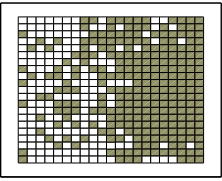

(h) $t=64 \mu \mathrm{s}$

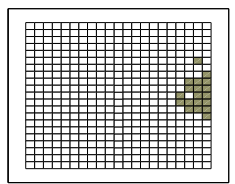

(c) $t=2 \mu \mathrm{s}$

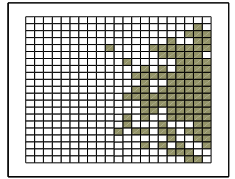

(f) $t=16 \mu \mathrm{s}$

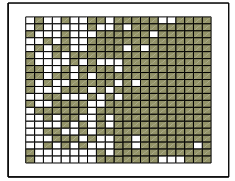

(i) $t=100 \mu \mathrm{s}$
Fig. 8: Time-course of glutamate-receptor binding on $21 \times 21$ grid of receptors with vesicle release site on the right edge.

than the simulation algorithm requiring position of neurotransmitters as random seeds in each time step. For comparison, the calculation of data with $N_{0}=3000$ and $P u=0.1$ plotted in both Fig. 5(a) and 5(b), took approximately 3 minutes on a PC for the deterministic code, while it took 1.5 hours on the same PC for each experiment of the simulation.

This significant improvement in speed allows us to analyze in greater detail the factors affecting peak amplitude of EPSP, which are directly related to $M_{b, \max }$. Therefore, in the following, we observe the effects of variations in different synaptic parameters such as the uptake probability, the number of neurotransmitters in a vesicle, the diffusion coefficient, the receptor density and the size of PSD, on $M_{b, \max }$ and $T_{p}$.

\section{Factors Affecting Maximum Bound Receptors}

1) Number of neurotransmitters per vesicle and presynaptic re-uptake: The variations in these quantities have direct effect on neurotransmitter concentration near PSD, and therefore, alter receptor binding dynamics significantly. High number of neurotransmitters $N_{0}$ per vesicle, or a low uptake probability $P_{u}$ results in a higher concentration of transmitters in the cleft, which, in turn, yields a faster binding rate and a higher value of $M_{b, \max }$ as shown in Fig. 6.

We see in Fig. 6(a) that PSD almost saturates for $N_{0}$ larger than 2000 transmitters per vesicle, but for lower quantities

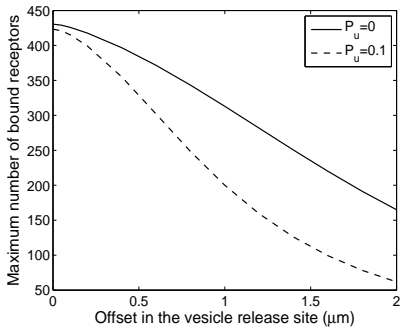

(a)

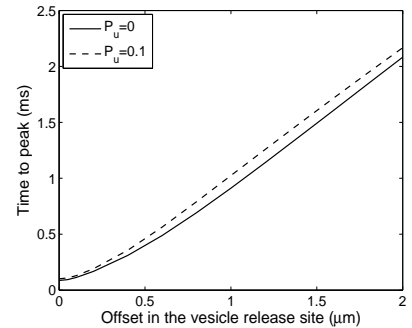

(b)
Fig. 9: Effect of offset in vesicle release site on (a) Maximum number of bound receptors (b) Time to peak.

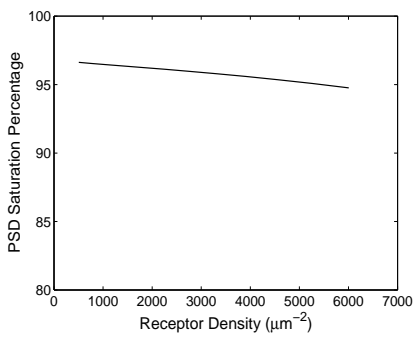

(a)

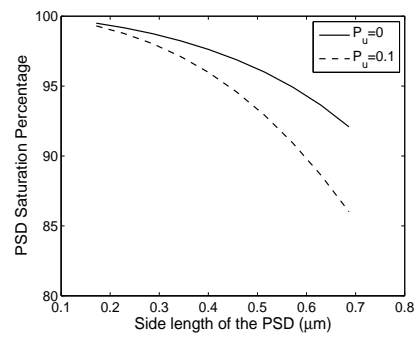

(b)
Fig. 10: PSD saturation with (a) receptor density (b) PSD size.

the variation in $M_{b, \max }$ becomes significant. Thus, a possible explanation for trial-to-trial variability of EPSP amplitude is that $N_{0}$ varies in the range $500-2000$. The large variance of $M_{b, \max }$ over this range also increases the neurons ability to encode and decode information.

Fig. 6(b) shows that even in case of no reflections from pre-synaptic boundary, i.e., $P_{u}=1$, the value of $M_{b, \max }$ is significantly high, at around 250 , which is more than half of the available receptors. This number is much higher for plausible values of $P_{u}$. This observation is in correspondence with the previous results of [20] that the pre-synaptic re-uptake has small effect on the peak amplitude of EPSP.

Time to reach maximum bound receptors $T_{p}$, somewhat counter-intuitively, is inversely related to $M_{b, \max }$, as seen in Fig. 7. After all, binding more receptors takes more time as expected. However, this is an observed phenomenon [32], and it is a recurring theme, in that, for any variation of a physiological parameter, which increases the transmitter concentration in the cleft, $M_{b, \max }$ increases, whereas $T_{p}$ decreases.

2) Vesicle release site: We first observe the effect of changing location of vesicle release site on $M_{b, \max }$. We add an offset of $200 \mathrm{~nm}$ in $\mathrm{x}$-direction in the original position of release site and obtain corresponding time-course of glutamate-receptor binding from Monte Carlo simulation as shown in Fig. 8. It is apparent that change in release site changes distribution and number of bound receptors reinforcing the argument that relocation of release site is one of the factors that change synaptic efficacy in subsequent trials.

For larger values of offset in vesicle release position the binding process takes significantly more time to reach its peak as shown in Fig. 9. As expected, moving release site away from receptor grid reduces the value of $M_{b, \max }$ and increases the time to peak. This can be explained by the same reasoning given for the changes caused by varying vesicle size and uptake probability, and the fact that, the further away from 
the PSD the transmitter release occurs, the lower will be the transmitter concentration near the PSD.

An important feature to observe from Fig. 9(a) is how the reducing effect of the uptake on $M_{b, \max }$ grows with the offset in the transmitter release location. This follows from the fact that, on a longer journey to the PSD the uptake mechanism captures more transmitters. This fact supports the notion that the synaptic channel noise due to contamination by transmitters from surrounding AZ-PSD pairs is small, which is in accordance with the findings of [20] that signaling at different glutamatergic synapses are independent of each other.

3) Receptor density and the size of the PSD: We simulate our model for different receptor densities keeping PSD area and other variables constant as mentioned in Table I such that number of unbound receptors in the given area increases. We observe in the Fig. 4 that a significant difference in $N_{0}$ and $M_{0}$ achieved high level of saturation of PSD, i.e., approximately $96 \%$. Therefore, we can intuitively predict that decreasing the difference in $N_{0}$ and $M_{0}$, obtains lower level of saturation. Thus, it is shown in Fig. 10(a) that the percentage of PSD saturation decreases with the increase in receptor density, however, the level of saturation does not fall significantly since the largest value for concentration in our simulation gives value of $M_{0}$ that is still quite lower than $N_{0}$. Therefore, we may observe drastic fall in saturation level for much higher values of receptor densities.

Furthermore, we just vary PSD area with fixed receptor density at 2750 receptors $/ \mu^{2}$, the reduction in saturation level is quite significant as shown in Fig.10(b). The reason for this effect is similar as for the effect caused by increasing off-set in vesicle release site. In both cases, distance between vesicle release site and a certain set of receptors is increased resulting in lower concentration of neurotransmitters around the receptors away from the release site, decreasing their binding probability, hence, we obtain lower level of saturation. This rate of reduction in saturation level is increased by increasing the uptake rate that further reduces number of neurotransmitters.

4) Diffusion coefficient: The diffusion coefficient $D$ encapsulates the rate of spread of glutamate molecules inside extracellular fluid medium of synaptic cleft, a higher value corresponding to faster spread. In literature, different values of $D$ has been used by different authors, all falling in the range $0.1-1 \mu \mathrm{m}^{2} / \mathrm{ms}$ [18]-[20]. As seen in Fig. 11, $M_{b, \max }$ decreases with increasing $D$ since with a larger $D$ the transmitters spread and leave the volume above the PSD faster.

\section{Error Probability}

Probability of error $P_{e}$ is a major performance measure of a communication channel. We simulate our model using parameters listed in Table I and determine $M_{b, \max }$ for different values of receptor density keeping PSD size constant. The response generated by bound receptors is detected by optimum receiver described in Section III.

Using (18), we evaluate $P_{e}$ as a function of postsynaptic receptor density for different values of vesicle release probabilities, as shown in Fig. 12. We keep incoming spike probability constant at $P(m=1)=0.7$. It can be observed

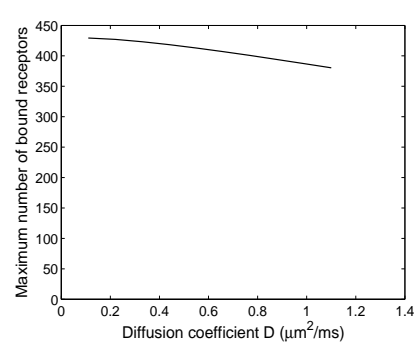

Fig. 11: $M_{b, \max }$ Vs $D$

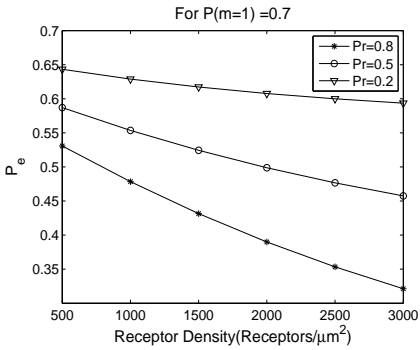

Fig. 12: $P_{e}$ Vs [AMPA]. that for a certain value of $P_{r}, P_{e}$ always reduces with the increase in receptor density. This result complies with the fact that given high probability of vesicle release, higher number of receptors on PSD would obtain higher value of $M_{b, \max }$ ensuring accurate detection, thus, reducing probability of error.

The advantage of the proposed model is that it enables us to analyze synaptic parameters on molecular level producing faster results than the conventional simulation models, as shown by the above discussion. Thus, we dedicated a subsection to analyze each important factor affecting the dynamics of the synaptic communication. Moreover, this study leads to the analysis of important parameters such as variation in vesicle size or receptor density that are responsible for synaptic plasticity in neurons and therefore, memory formation.

\section{CONCLUSION}

In this article, we investigated one of the most advanced mechanism of molecular communication that exists in nature. We focused on the point-to-point synaptic channel in hippocampal neuron and introduced a novel mathematical formulation of neurotransmitters' diffusion in synaptic cleft that encompasses the effects of geometrical structure of synapse and re-uptake phenomenon. As a result of this mathematical achievement we have developed a deterministic code, which calculates the expected behavior of synaptic molecular communication channel. The predictions of this code are successfully verified by a corresponding Monte Carlo simulation. This is one of the main contributions of this paper, as the code developed takes much less time for the same task compared to the Monte Carlo approach.

Moreover, we observed the effects of variations in various synaptic parameters on number of maximum bound receptors and time to reach this maximum, which together determine EPSP profile. The results obtained in this analysis comply with the outcomes of existing physiological studies and simulations in literature. We also discussed the possible causes of trial-totrial variability in peak amplitude of EPSP. Eventually, we performed communication theoretical analysis of our model by evaluating error probability in output detection.

\section{REFERENCES}

[1] I. F. Akyildiz, F. Brunetti, and C. Blázquez, "Nanonetworks: A new communication paradigm," Computer Networks, vol. 52, no. 12, pp. 2260-2279, 2008.

[2] T. Nakano, M. J. Moore, F. Wei, A. V. Vasilakos, and J. Shuai, "Molecular communication and networking: Opportunities and challenges," IEEE Trans. NanoBiosci., vol. 11, no. 2, pp. 135-148, 2012.

[3] O. B. Akan, H. Ramezani, T. Khan, N. A. Abbasi, and M. Kuscu, "Fundamentals of molecular information and communication science," Proc. of the IEEE, to be published, 2016. 
[4] D. Malak and O. B. Akan, "Molecular communication nanonetworks inside human body," Nano Commun. Netw., vol. 3, no. 1, pp. 19-35, 2012.

[5] D. Malak, H. Ramezani, M. Kocaoglu, and O. B. Akan, "Diversity in diffusion-based molecular communication channel with drift," in Proc. ICC. IEEE, 2016, pp. 1-6.

[6] M. Kuscu and O. B. Akan, "On the physical design of molecular communication receiver based on nanoscale biosensors," IEEE Sensors Journal, vol. 16, no. 8, pp. 2228-2243, 2016.

[7] — " "On the capacity of diffusion-based molecular communications with sinw fet-based receiver," in Proc. of EMBC. IEEE, 2016, pp. 3043-3047.

[8] E. Balevi and O. B. Akan, "A physical channel model for nanoscale neuro-spike communications," IEEE Trans. Commun., vol. 61, no. 3, pp. 1178-1187, 2013.

[9] D. Malak and O. B. Akan, "A communication theoretical analysis of synaptic multiple-access channel in hippocampal-cortical neurons," IEEE Trans. Commun., vol. 61, no. 6, pp. 2457-2467, 2013.

[10] N. A. Abbasi and O. B. Akan, "A queueing-theoretical delay analysis for intra-body nervous nanonetwork," Nano Commun. Netw., vol. 6, no. 4, pp. 166-177, 2015.

[11] D. Malak and O. B. Akan, "Communication theoretical understanding of intra-body nervous nanonetworks," IEEE Communications Magazine, vol. 52, no. 4, pp. 129-135, 2014.

[12] H. Ramezani and O. B. Akan, "Synaptic channel model including effects of spike width variation," in Proc. of ACM NANOCOM, 2015, p. 11.

[13] N. C. Danbolt, "Glutamate uptake," Prog. Neurobiol., vol. 65, no. 1, pp. 1-105, 2001.

[14] P. Dayan and L. Abbott, "Theoretical neuroscience: computational and mathematical modeling of neural systems," Journal of Cognitive Neuroscience, vol. 15, no. 1, pp. 154-155, 2003.

[15] A. Hodgkin and A. Huxley, "Propagation of electrical signals along giant nerve fibres," Proc R Soc Lond B Biol Sci., pp. 177-183, 1952.

[16] M. Veletić, P. A. Floor, Z. Babić, and I. Balasingham, "Peer-to-peer communication in neuronal nano-network," IEEE Trans. Commun., vol. 64, no. 3, pp. 1153-1166, 2016.

[17] A. Manwani and C. Koch, "Detecting and estimating signals over noisy and unreliable synapses: information-theoretic analysis," Neural comput., vol. 13, no. 1, pp. 1-33, 2001.

[18] N. Agmon and A. L. Edelstein, "Collective binding properties of receptor arrays." Biophys. J., vol. 72, no. 4, p. 1582, 1997.

[19] F. Ventriglia and V. Di Maio, "A brownian simulation model of glutamate synaptic diffusion in the femtosecond time scale," Biol. Cybern., vol. 83, no. 2, pp. 93-109, 2000.

[20] K. M. Franks, T. M. Bartol, and T. J. Sejnowski, "A monte carlo model reveals independent signaling at central glutamatergic synapses," Biophysical journal, vol. 83, no. 5, pp. 2333-2348, 2002.

[21] K. M. Franks, C. F. Stevens, and T. J. Sejnowski, "Independent sources of quantal variability at single glutamatergic synapses," J.Neurosci., vol. 23 , no. 8, pp. 3186-3195, 2003.

[22] C. F. Stevens and Y. Wang, "Facilitation and depression at single central synapses," Neuron, vol. 14, no. 4, pp. 795-802, 1995.

[23] E. L. Cussler, Diffusion: mass transfer in fluid systems. Cambridge university press, 2009.

[24] P. Dayan and L. F. Abbott, Theoretical Neuroscience: Computational and Mathematical Modeling of Neural Systems. MIT Press, 2001.

[25] L. P. Savtchenko and D. A. Rusakov, "The optimal height of the synaptic cleft," Proc Natl Acad Sci U S A, vol. 104, no. 6, pp. 1823-1828, 2007.

[26] D. A. Rusakov, L. P. Savtchenko, K. Zheng, and J. M. Henley, "Shaping the synaptic signal: molecular mobility inside and outside the cleft," Trends Neurosci., vol. 34, no. 7, pp. 359-369, 2011.

[27] T. A. Nielsen, D. A. DiGregorio, and R. A. Silver, "Modulation of glutamate mobility reveals the mechanism underlying slow-rising ampar epses and the diffusion coefficient in the synaptic cleft," Neuron, vol. 42, no. 5, pp. 757-771, 2004.

[28] J. Montes, J. M. Peña, J. DeFelipe, O. Herreras, and A. Merchan-Perez, "The influence of synaptic size on ampa receptor activation: A monte carlo model," PloS one, vol. 10, no. 6, p. e0130924, 2015.

[29] J. Clements, "Transmitter timecourse in the synaptic cleft: its role in central synaptic function," Trends Neurosci., vol. 19, no. 5, pp. 163 171, 1996.

[30] G. Tong and C. E. Jahr, "Block of glutamate transporters potentiates postsynaptic excitation," Neuron, vol. 13, no. 5, pp. 1195-1203, 1994.

[31] C.-M. Tang, M. Margulis, Q.-Y. Shi, and A. Fielding, "Saturation of postsynaptic glutamate receptors after quantal release of transmitter," Neuron, vol. 13, no. 6, pp. 1385-1393, 1994.

[32] R. Sayer, M. Friedlander, and S. Redman, "The time course and amplitude of epsps evoked at synapses between pairs of ca3/ca1 neurons in the hippocampal slice," J. Neurosci., vol. 10, no. 3, pp. 826-836, 1990.

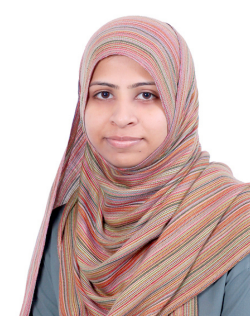

nanonetworks
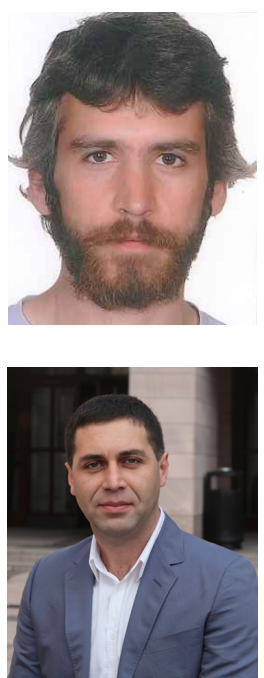

Tooba Khan [S'17] received the B.E. and M.S. degrees in Telecommunications Engineering from NED University of Engineering and Technology, Pakistan, in 2009 and 2012, respectively. She is currently working towards the Ph.D. degree in Electrical and Electronics Engineering at Koc University, Istanbul, Turkey. She is a Research Assistant in the Next-Generation and Wireless Communication Laboratory (NWCL), Koc University. Her current research interests include molecular communications and intrabody

Bilgesu A. Bilgin []M'17] received his B.S. degree in Electrical and Electronics engineering from Middle East Technical University in 2008, M.Sc. and Ph.D. degrees in mathematics from Koc University, in 2011 and 2015, respectively. He is currently carrying out his research as a postdoctoral fellow in Next-generation and Wireless Communications Laboratory. His research interests include molecular communication, intrabody nanonetworks, partial differential equations and dynamical systems.

Ozgur B. Akan [M'00-SM'07-F'16] received PhD degree from the School of Electrical and Computer Engineering, Georgia Institute of Technology, USA, in 2004. He is currently with the Electrical Engineering Division, Department of Engineering, University of Cambridge, UK and the Department of Electrical and Electronics Engineering, Koc University, Istanbul, Turkey. His research interests include wireless, nano, molecular communications, and Internet of Everything. 